\title{
The role of apparent motion cues in orientation masking
}

\author{
PETER WENDEROTH and TIM TYLER \\ University of Sydney, Sydney, New South Wales 2006, Australia
}

\begin{abstract}
It has been reported that the tuning function relating masking of a vertical line to the orientation of a masking line differs in form depending upon whether the mask is presented with the target (simultaneous masking) or before it (forward masking). From such evidence, it might be concluded that different mechanisms are implicated. Lovegrove (1976) suggested that lateral inhibition mediates the simultaneous effect, whereas postexcitatory suppression (adaptation) mediates the successive effect. The six experiments reported here show that apparent motion cues reduce the effectiveness of successive masking when single-line stimuli are used, but that when such motion cues are eliminated, the two tuning functions are similar in form. It is, thus, unnecessary to invoke separate mechanisms in the two cases.
\end{abstract}

The detection threshold of a vertical target line is raised by either the simultaneous or successive presentation of another (masking) line if the mask is at or near vertical (Houlihan \& Sekuler, 1968). Lovegrove (1976) compared the tuning range of masking in the simultaneous and successive cases: The target line was always vertical, while the mask's orientation was varied in $15^{\circ}$ steps from vertical $\left(0^{\circ}\right)$ to horizontal $\left(90^{\circ}\right)$. In the simultaneous case, Lovegrove found that the target's threshold was increased significantly by all mask orientations. However, the successive masking paradigm, in which the mask preceded the target (forward masking), produced significant threshold increase only when the mask and target were both vertical. According to Lovegrove, this difference in the breadth of tuning of the two types of masking implies that different mechanisms operate in the two cases. He postulated that the broader simultaneous masking function reflects lateral inhibitory processes (Carpenter \& Blakemore, 1973; Tolhurst \& Thompson, 1975) while successive masking reflects the operation of postexcitatory suppression (Barlow \& Hill, 1963; Over, 1971) or adaptation (Coltheart, 1971b).

Inspection of Lovegrove's data suggests that it may be unnecessary to invoke different mechanisms in the two cases. Although Lovegrove did not include a vertical mask condition in his simultaneous masking experiment, comparison of the simultaneous and successive functions in the common mask orientation range from $15^{\circ}$ to $90^{\circ}$ indicates that the two functions have similar shapes but that the simultaneous function is

This research was supported, in part, by the Australian Research Grants Committee, Grant A74/15177 to the first author, and by a University of Sydney Research Grant. We thank Warren Davies who modified the tachistoscope. simply shifted upwards by a constant amount. This could come about if similar mechanisms operate in the two cases and if additional cues are available in the successive paradigm which are relatively independent of mask orientation but which allow the observer to overcome the masking effect to some extent. In particular, when two lines at neighboring orientations are flashed with the interstimulus interval (ISI) of $10 \mathrm{msec}$ which Lovegrove used, observers often report stroboscopic motion: the tilted mask followed by the vertical target are seen as a single line rotating from a tilted position to vertical. During the course of our investigation, an independent study reported data consistent with this hypothesis of motion cues in successive masking. Carlson and Mayzner (1977) noted in the case of backward masking that "If the mask was preceded by a horizontal line, it was seen as a group of horizontal vectors moving centrifugally toward the top and bottom edges of the mask.... These target-induced alterations occurred when there was considerable target suppression: Target recognition was only $20 \%$ accurate when the above observations were noted [p. 227] .... Our subjects reported that, although they could not identify the targets per se, they could have inferred their orientation and geometry on the basis of mask-movement cues [p. 229]."'

Six experiments are reported here and they had two broad aims. First, we repeated Lovegrove's experiments under conditions not identical to his but as similar as we could arrange, with the aim of adducing evidence relevant to the role of apparent motion cues (Experiments 1 to 3). Second, we varied stimulus parameters so as to eliminate apparent motion as a possible cue, in order then to compare the tuning characteristics of simultaneous and successive masking in the orientation domain (Experiments 4 to 6$)$. 


\section{EXPERIMENTS 1 TO 3}

First, we attempted to repeat Lovegrove's experiments on simultaneous masking (Experiment 1), successive masking (Experiment 2), and disinhibition in successive masking (Experiment 3 ).

\section{Method}

Subjects. In each experiment, a previously untested group of five students from an introductory psychology course volunteered. All had normal or corrected vision and were unaware of our aims and methods.

Apparatus. Three fields of a Gerbrands four-channel Model 4A tachistoscope were used to present the stimuli, which were single white lines on biack backgrounds, positioned so that their centers coincided. On noise trials, a homogeneous dark field of the same space-average luminance as the target field was presented instead of the target line and a similar field with the same space-average luminance as the mask field was used to obtain a baseline threshold for masking by a homogeneous field. In the first experiment, the fixation area was defined by intermittent presentation of the target stimulus alone; and although no subjects experienced difficulty in maintaining fixation, a dim fixation point was provided in the fourth field in subsequent experiments. Table 1 provides a comparison of the stimulus parameters we used and those of Lovegrove. Luminances necessarily were less in our experiments because of the limited range available with Gerbrands as compared with Scientific Prototype tachistoscopes.

Lovegrove (1976) stated that the 300 -msec mask and the briefly flashed target were "simultaneously presented" in his first experiment, which we took to mean that their onsets coincided. In Experiments 2 and 3, mask onset preceded target onset by 20 msec.

Apart from temporal parameters, only the mask stimuli varied over the three experiments. In Experiment 1, a single masking line was oriented $15^{\circ}, 30^{\circ}, 45^{\circ}, 60^{\circ}, 75^{\circ}$, or $90^{\circ}$ clockwise of vertical. The same masks were used in Experiment 2 except that an additional, vertical mask was included. In Experiment 3, the mask contained two lines, one always vertical and the other oriented $15^{\circ}$, $30^{\circ}, 45^{\circ}, 60^{\circ}, 75^{\circ}$, or $90^{\circ}$, arranged so that the two masking lines crossed at their centers.

Procedure. A blockwise tracking procedure was used (Campbell, 1963), identical to that used by Lovegrove (1976): Thresholds were obtained for each mask orientation and each subject was tested under each condition. For a given mask orientation, a block of 12 target trials $[6$ signal trials and 6 noise (blank) trials, randomly interleaved] was initially given at a fixed exposure level of the target. The target duration was varied in ratio steps (e.g., 1, 2, 4, 8, 16 ... msec) over blocks until the observer achieved $75 \%$ accuracy in a single block or bracketed this value in successive blocks. The order of testing under experimental conditions (mask lines present)

Table 1

Comparison of Stimulus Parameters Used by Lovegrove (1976) and in Experiment 1

\begin{tabular}{lll}
\hline & \multicolumn{2}{c}{ Study } \\
\cline { 2 - 3 } \multicolumn{1}{c}{ Stimulus Parameter } & $\begin{array}{c}\text { Lovegrove } \\
(1976)\end{array}$ & Experiment 1 \\
\hline Circular Field Diameter & $4^{\circ} 30^{\prime}$ & $4^{\circ} 30^{\prime}$ \\
Target Line Dimensions & $1^{\circ} 30^{\prime} \times ?$ & $1^{\circ} 30^{\prime} \times .8^{\prime}$ \\
Target Space Average Luminance & $1.1 \mathrm{~cd} / \mathrm{m}^{2}$ & $.34 \mathrm{~cd} / \mathrm{m}^{2}$ \\
Target Contrast & .6 & .67 \\
Mask Line Dimensions & $2^{\circ} 30^{\circ} \times ?$ & $2^{\circ} 30^{\prime} \times .8^{\prime}$ \\
Mask Space Average Luminance & $4.4 \mathrm{~cd} / \mathrm{m}^{2}$ & $1.1 \mathrm{~cd} / \mathrm{m}^{2}$ \\
Target/Mask Luminance Ratio & .25 & .31 \\
Mask Duration & $300 \mathrm{msec}$ & $300 \mathrm{msec}$ \\
\hline
\end{tabular}

and control conditions (masking by homogeneous field) was randomly determined.

Presumably in accordance with Lovegrove's procedure, no feedback was given and the subjects were not instructed concerning signal presentation probability. A tap was given as a warning $1 \mathrm{sec}$ before each trial and the subject responded "yes" (target present) or "no." Head movement was restrained sufficiently by a viewing mask into which the subjects pressed their faces, and viewing was always right-eye monocular. The intertrial interval was approximately $5 \mathrm{sec}$, with longer breaks between blocks of 12 trials.

The subjects were read common instructions, ending with an appeal for "a running commentary on anything which strikes you as strange or interesting in the display." After between three to six mask orientations had been tested, the subjects were asked a standard question: "What are you using as a criterion for saying "yes'?" If this was not understood, the question was rephrased to: "What has to happen before you say 'yes'?"

\section{Results}

The results of Experiments 1 to 3 are shown in Figures 1 to 3 , together with Lovegrove's data for comparison. In each case, the masking ratio on the ordinate is given by the target duration for $75 \%$ correct responses on experimental trials, divided by the duration for $75 \%$ correct on control trials. Absence of masking would result in a unit ratio (dashed lines).

Generally, our results resembled those of Lovegrove. The target durations at threshold with homogeneousfield masking were $1.40,1.50$, and $1.8 \mathrm{msec}$ in $\mathrm{Ex}$ periments 1 to 3 , respectively (Lovegrove: $1.40,1.49$, and $1.60 \mathrm{msec}$ ). In Experiment 1, we obtained a significant overall effect of mask orientation $[F(5,10)=$ $16.52, \mathrm{p}<.01$ ], and protected $t$ tests (Scheffé, 1953) indicated that all ratios were greater than 1.0, except those at $60^{\circ}$ and $75^{\circ}$. Lovegrove obtained significant masking at all orientations. The half-width of our masking function $\left(15^{\circ}-30^{\circ}\right)$ appears less than Lovegrove's $\left(45^{\circ}-60^{\circ}\right)$, d.e to the much greater masking we obtained at $15^{\circ}$.

In Experiment 2, there was also a significant overall effect of mask orientation $[F(6,24)=30.24$, $\mathrm{p}<.01]$. Like Lovegrove, we obtained smaller masking ratios in Experiment 2 than in Experiment 1 and our interpolated half-width of $10.5^{\circ}$ was similar to Lovegrove's and, indeed, to that obtained by Kulikowski (1972), namely $7.4^{\circ}$. However, protected $t$ tests showed that masking ratios exceeded 1.0 at $0^{\circ}, 15^{\circ}, 30^{\circ}$, and $60^{\circ}$, whereas Lovegrove obtained significant masking only at $0^{\circ}$.

Unlike Lovegrove, we did not obtain a significant effect of orientation of the variable mask component in Experiment $3[\mathrm{~F}(6,24)=1.69, \mathrm{p}>.05]$ although subsequent $t$ tests indicated that all masking ratios exceeded 1.0. The logic of Experiment 3 was that the tilted mask should inhibit the vertical mask, thus releasing the target from inhibition by the latter. Lovegrove did find such disinhibition at all orientations except $45^{\circ}$, but in our experiment, more masking occurred whenever a second, tilted mask line was present, compared to the effect when it was absent. 


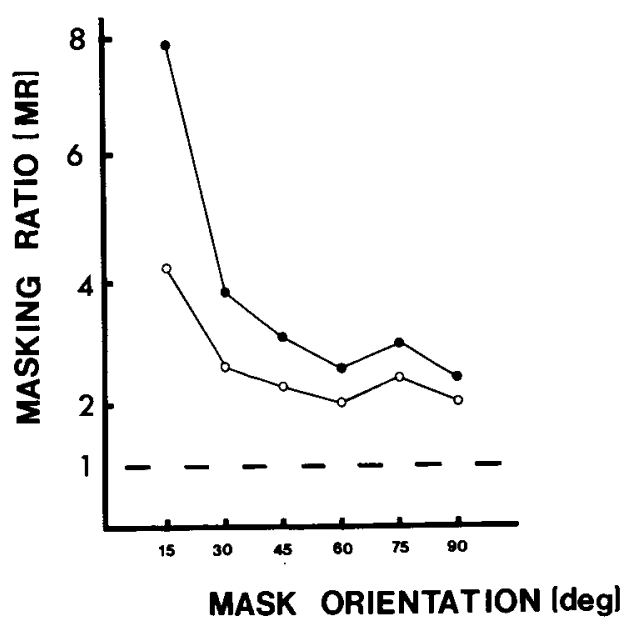

Figure 1. Simultaneous masking ratios as a function of mask orientation, obtained in Experiment 1 (filled circles) and by Lovegrove (open circles).

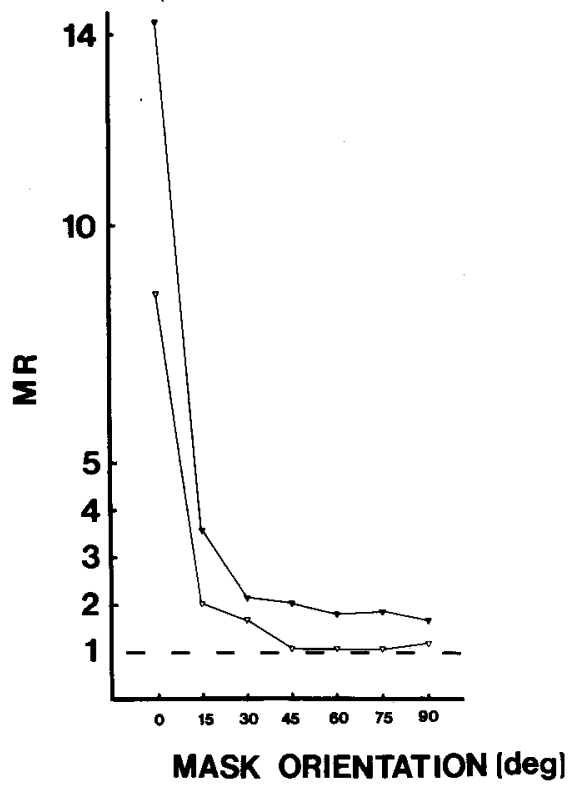

Figure 2. Successive masking ratios as a function of mask orientation, obtained in Experiment 2 (filled triangles) and by Lovegrove (open triangles).

The subjects' responses to the request for verbal reports of judgmental criteria suggested a reason for the reduced masking in Experiment 2 compared to Experiment 1, and for the significant masking with no main effect of orientation in Experiment 3. Whereas Experiment 1 subjects seemed puzzled by the question we asked and simply repeated the instructions (viz, "I said 'yes' when I saw the vertical line"), all subjects in Experiment 2 reported apparent motion and all admitted responding "yes" when such motion was detected and "no" when it was not detected, whether the target line was clearly seen or not. This finding is consistent with that of Kolers
(1972) who reported apparent motion between two disks even when the first was masked by an annulus; and it is also consistent with recent analysis of visual processing into parallel components mediated by sustained and transient channels (Breitmeyer \& Ganz, 1976).

The subjects in Experiment 2 all reported using apparent motion as a cue, but not consistently. In Experiment 3, on the other hand, they not only reported using the cue but described a different kind of motion, which, they said, occurred on progressively more trials as the experiment proceeded. In Experiment 2 , the tilted mask appeared to rotate to the vertical target position for all mask orientations other than $0^{\circ}$; at $0^{\circ}$, some subjects noted that the longer mask ( $2^{\circ} 30^{\prime}$ in length) seemed to "shrink into" the shorter, $1^{\circ} 30^{\prime}$ target. Because a vertical mask component was always present in Experiment 3, the subjects reported rotation and then shrinkage in all conditions. A possible reason for the increase in reports of shrinkage motion over trials in Experiment 3 can be found in Kolers' $(1972$, p. 118) report that "when two lines forming an angle were alternated many times ... good motion continued to be seen for a few trials even when one of the lines was suddenly eliminated from the display [italics ours]."

Consistent with this hypothesis regarding the subjects' strategies in Experiment 3 is the fact that the ratio of false alarms to misses was low consistently in Experiment 1, in which motion cues were not available. The ratio was also low, although it peaked slightly in the middle blocks in Experiment 2, in which subjects reported using motion cues, but not consistently. In Experiment 3, however, the ratio steadily increased over trial blocks, for all subjects,

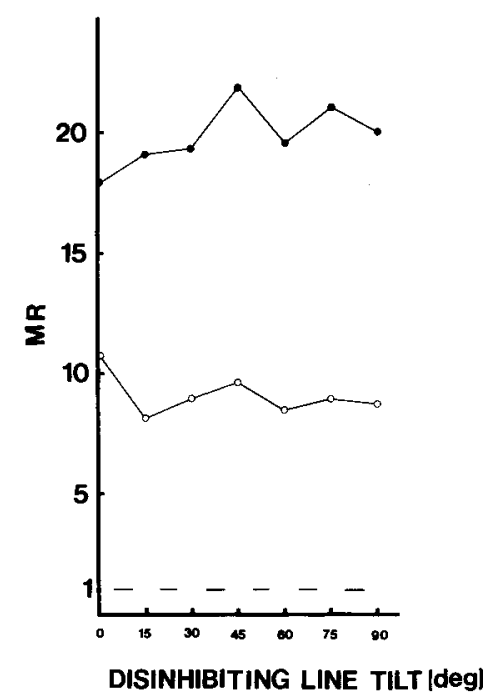

Figure 3. Masking ratios as a function of disinhibiting line orientation, obtained in Experiment 3 (filled circles) and by Lovegrove (open circles). 


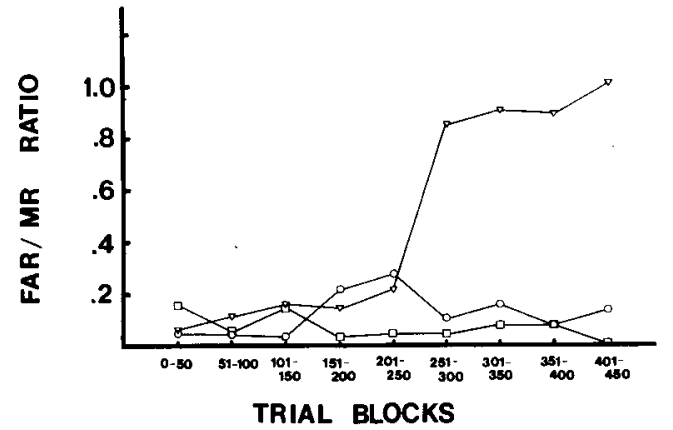

Figure 4. Ratio of false-alarm rate to miss rate over blocks of 50 trials in Experiments 1 (squares), 2 (circles), and 3 (triangles).

resulting in large masking ratios. Thus, the use of this inappropriate cue of apparent shrinkage may have concealed any real effect of mask orientation. The false-alarm-rate to miss-rate ratio (FAR/MR) over trial blocks is shown for each experiment in Figure 4.

We also tested one practised observer under some of the conditions of Experiments 2 and 3. Her ratings of motion clarity confirmed the presence of motion in both paradigms and as well as the particular salience of it under Experiment 3 conditions.

\section{EXPERIMENTS 4 TO 6}

The results of the first three experiments indicated that it would be necessary to eliminate apparent motion cues from the successive masking task before any real comparison between the tuning ranges of simultaneous and successive masking could be made.

\section{Method}

Various techniques were tried, including variation in ISI, but all were unsuccessful in eliminating apparent motion. The method eventually selected was to use a target line but a masking grating: Both Houlihan and Sekuler (1968) and Sekuler (1965) used these stimuli; and Sekuler (1965) reported absence of apparent motion in such a backward masking paradigm. In addition, Weisstein and Bisaha (1972) showed that gratings mask bars and vice versa and Sullivan, Georgeson, and Oatley (1972) demonstrated that targetline width is not a critical determinant of masking of aperiodic stimuli by periodic stimuli.

Experiments 4 to 6, then, were replications of the first three experiments, with the single difference that the mask was a Letratone square-wave grating of spatial frequency $5.6 \mathrm{cycles} / \mathrm{deg}$ with luminance and contrast equal to that of the masking stimuli in Experiments 1 to 3 . The white target line was positioned so that it was superimposed on a black bar of the grating which reduced the subjects' confusion when both mask and target were vertical in the simultaneous masking condition. In each experiment, five new subjects were tested.

\section{Results}

The results of Experiments 4 (simultaneous masking) and 5 (successive masking) are shown in Figure 5. In the simultaneous case, the overall effect of orien- tation was significant $[F(6,24)=16.26, p<.01]$ and protected $t$ tests indicated that the masking ratio was significantly greater than 1.0 at all orientations except $90^{\circ}$. In the successive case, the overall effect of mask orientation was also significant $[F(6,24)$ $=21.53, \mathrm{p}<.01]$ and all ratios were significantly greater than 1.0 except those at $60^{\circ}$ and $90^{\circ}$. Clearly, the functions are very similar, with half-widths between $30^{\circ}$ to $45^{\circ}$; the difference is that the simultaneous function lies about 1.0 to 1.5 ratio units above the successive function.

The disinhibition function obtained in Experiment 6 is shown in Figure 6.

In the absence of apparent motion cues, it is clear that, relative to the normalized masking ratio of 1.0 that was obtained when the disinhibitory grating coincided with the masking grating at $0^{\circ}$, less masking occurred for all other orientations of the disinhibitory grating. The overall effect of the disinhibitory grating's orientation was significant $[F(6,24)$ $=5.0, p<.05]$, and $t$ tests showed that significantly less masking than at $0^{\circ}$ occurred at orientations of $15^{\circ}, 30^{\circ}, 75^{\circ}$, and $90^{\circ}$.

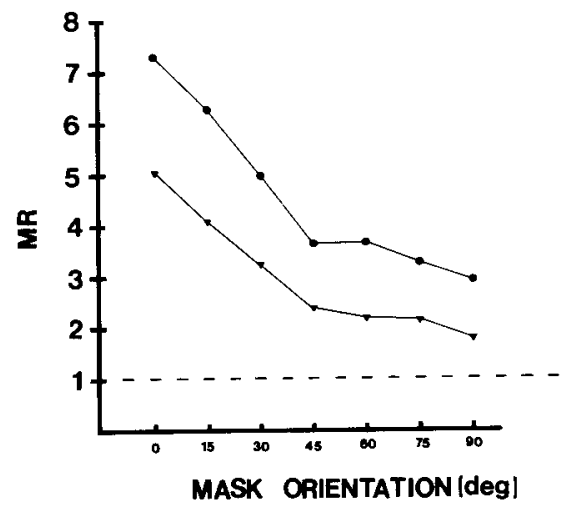

Figure 5. Masking ratios as a function of mask orientation obtained in Experiment 4 with simultaneous masking (circles) and Experiment 5 with successive masking (triangles).

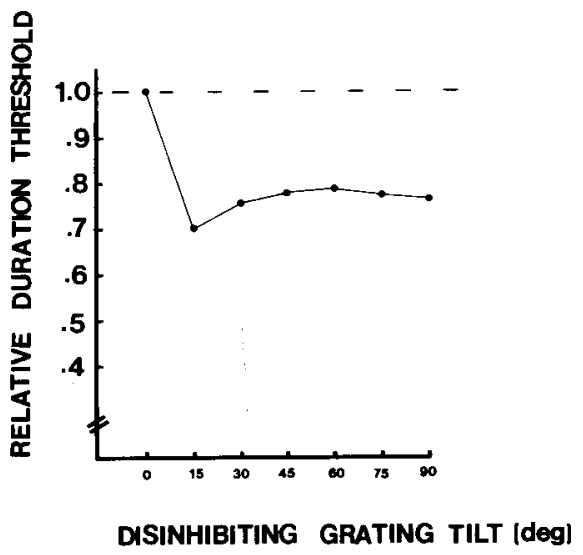

Figure 6. Relative duration threshold for target line as a function of disinhibiting grating orientation. 


\section{DISCUSSION}

The experiments reported here do not discriminate between the possible roles of lateral inhibitory processes and postexcitatory suppression in orientation masking. However, the data do suggest that different mechanisms need not be invoked to account for successive and simultaneous masking effects.

One parsimonious possibility is that the masking stimulus propagates orientation-selective lateral inhibition and that such inhibitory effects are strongest when the target and mask onsets coincide or almost coincide. When the mask precedes the target by $20 \mathrm{msec}$ so that, in our case and Lovegrove's, the mask onset precedes the target onset by $320 \mathrm{msec}$, the inhibition propagated by the mask decays somewhat and is relatively weaker by the time the target is presented. Hence, the masking effect is weaker but the tuning function is similar in form to that obtained with simultaneous masking. This kind of proposal would account for the near-parallel functions in Figure 5.

Finally, it should be stated explicitly that the conclusion we wish to draw from these experiments is only that Lovegrove's data (and ours) do not require the postulate that different mechanisms underlie simultaneous and successive masking: the data can be accounted for in terms of a single mechanism. However, although we have chosen to refer to that mechanism as "lateral inhibition," we concede that this is not the only possible candidate. For example, there has been debate in the literature as to whether the tilt aftereffect is due to "adaptation" or to the prolonged effects of "lateral inhibition" (e.g., Blakemore, Carpenter, \& Georgeson, 1971; Coltheart, 1971a). Thus, Sekuler and Littlejohn's (1974) finding that the aftereffect asymptotes after only $18 \mathrm{msec}$ of inspection led them to reject Coltheart's (1971b) proprosal that prolonged adaptation accounts for the effect. But this level of debate may not be productive, since it is only necessary to speak of "short-term adaptation" to account for these data. A parallel can be drawn here between the "inhibition-adaptation" debate and the current "neural adaptation-classical conditioning" debate in relation to the McCullough effect (e.g., Skowbo, Timney, Gentry, \& Morant, 1975). In the latter case, the fact that the effect persists for a long time as well as other data have led some to propose a learning model. On the other hand, the effect's failure to transfer interocularly and its failure to occur in the depth domain when Julesz patterns are used, as well as other data, have led some to reject the learning account. However, such debate is essentially confined to the verbal level, because one can speculate about "long-term neural adaptation" which involves synaptic changes or, equally easily, about "conditioning" which occurs only in monocular, color-selective visual channels.
A similar point was made by Creutzfeldt and Heggelund (1975) when they noted that their observations of decreased responsiveness in single neurons of the cat cortex could be explained either in terms of "adaptation" of excitatory connections or in terms of facilitation of inhibitory networks. Thus, although we probably favor the "lateral inhibition" account of the masking effects reported here, as well as the tilt illusion and aftereffect and related phenomena, we accept that others may wish to use the term "adaptation"; but we agree with Sekuler and Littlejohn (1974) that backward masking and aftereffect seem more easily to be explicable in "inhibition" than in "adaptation" terminology. In the end, the critical test of the mechanisms underlying the various aftereffect, adaptation, and masking phenomena probably will require direct physiological observations of the kind which are already beginning to be reported (e.g., Benevento, Creutzfeldt, \& Kuhnt, 1972; Blakemore \& Tobin, 1972; Nelson \& Frost, 1978).

\section{REFERENCES}

BARLow, H. B., \& HrLl, R. Evidence for a physiological explanation of the waterfall phenomenon and figural aftereffects. Nature, 1963, 200, 1434.

Benevento, L. A., Creutzfeldt, O. D., \& Kuhnt, U. Significance of intracortical inhibition in the visual cortex. Nature, 1972, 238, 124-126.

Blakemore, C., Carpenter, R., \& Georgeson, M. Lateral thinking about lateral inhibition. Nature, 1971, 234, 418-419.

Blakemore, C., \& Tobin, E. Lateral inhibition between orientation detectors in the cat's visual cortex. Experimental Brain Research, 1972, 15, 439-440.

BREITMEYER, B., \& GANz, L. Implications of sustained and transient channels for theories of visual pattern masking, saccadic suppression and information processing. Psychological Review, 1976, 83, 1-36.

Campbell, R. A. Detection of a noise signal of varying duration. Journal of the Acoustical Society of America, 1963, 35, 1732-1737.

Carlson, K., \& MaYzner, M. S. A reassessment of target-mask interaction in visual backward masking. Bulletin of the Psychonomic Society, 1977, 9, 227-229.

Carpenter, R. H. S., \& Blakemore, C. Interactions between orientations in human vision. Experimental Brain Research, 1973, 18, 287-303.

Coltheart, M. Is there lateral inhibition in the visual cortex? Nature, 1971, 231, 256. (a)

Coltheart, M. Visual feature analyzers and aftereffects of tilt and curvature. Psychological Review, 1971, 78, 114-121. (b)

Creutzfeldt, O., \& Heggelund, P. Neutal plasticity in visual cortex of adult cats after exposure to visual patterns. Science, $1975,188,1025-1027$.

Houlihan, K., \& Sekuler, R. W. Contour interactions in visual masking. Joumal of Experimental Psychology, 1968, 77, $281-285$.

Kolers, P. A. Aspects of motion perception. Oxford: Pergamon, 1972.

KULIKowsKI, J. J. Orientational selectivity of human binocular and monocular vision revealed by simultaneous and successive masking. Joumal of Physiology, 1972, 226, 67-69P.

LOVEgROVE, W. Inhibition in simultaneous and successive contour interaction in human vision. Vision Research, 1976, 16. 1519-1521. 
Mollon. J. D.. \& Polden. P. G. On the time constants of tachistoscopes. Quatterly Jounal of Experimental Psychology, 1978. 30. 555-568.

Nelson, J. I., \& Frost, B. J. Orientation-selective inhibition from beyond the classic visual receptive field. Brain Research, 1978, 139. 359-365.

OVER, R. Comparisons of normalization theory and neural enhancement explanation of negative after-effects. Psychological Bulletin, 1971, 75, 225-243.

SchefFE, H. A method for judging all contrasts in the analysis of variance. Biometrika, 1953, 40, 87-104.

SEkuler, R. Spatial and temporal determinants of visual backward masking. Journal of Experimental Psychology, 1965, 70, 401-406.

SeKUlER, R., \& LitTlejohn, J. Tilt after-effect following very brief exposures. Vision Research, 1974, 14, 151-152.

Skowbo, D., Timney, B. N., Gentry, T. A., \& Morant, R. B. McCollough effects: Experimental findings and theoretical accounts. Psychological Bulletin, 1975, 82, 497-510.

Sullivan, G. D., Georgeson, M. A., \& Oatley, K. Channels for spatial frequency selection and the detection of single bars by the human visual system. Vision Research, 1972, 12, 383-394.

Tolnurst, D. J., \& Thompson, P. G. Orientation illusions and aftereffects: Inhibition between channels. Vision Research, 1975 , 15. $967-972$.

WEISSTEIN, N., \& BisAHA, S. Gratings mask bars and bars mask gratings: Visual response to aperiodic stimuli. Science, 1972, 176, 1047-1049.

\section{NOTE ADDED IN PROOF}

Recently, Mollon and Polden (1978) have demonstrated, devastatingly and with wit, what many researchers knew but had not quantified: that almost all commercial tachistoscopes' time constants are deplorable. Mollon and Polden also show how some reported findings could be artifacts of lamp characteristics. However, it seems unlikely that the present data are explicable this way: varying mask orientation is independent of lamp characteristics. Nevertheless, in view of Mollon and Polden's work, at the very least one would hesitate to use the present data in a quantitative model.

(Received for publication October 6, 1978; revision accepted January 20,1979.) 